Causeret J., Hugot D., Goulas-Scholler C., Mocquot G., 1961. Evolution de la teneur du lait stérilisé en riboflavine et en vitamine A au cours d'une conservation de longue durée. Ann. Techn. agric., 10, 289-300.

Hugot D., Lhuissier M., Causeret J., 1962. Effet protecteur des bouteilles de verre ambré contre les pertes de riboflavine et de vitamine $\mathrm{A}$ dues à l'exposition du lait stérilisé à la lumière. Ann. Techn. agric., 11, 145-151.

JosEPHSON D. V., 1946. Some observations regarding the effect of various wavelenghts of light on the riboflavin content and flavor of milk. $J$. Dairy Sci., 29, 508-510.

Lhuissier M., Hugot D., Biette E., 1962. Etude vitaminologique du lait "upérisé " conditionné aseptiquement en récipients Tetra Pak : influence de la conservation. L'Industrie laitière, 194, 319-320.

Snell E. E., Strong F. M., 1939. A microbiological assay for riboflavin. Industri. Engng. Chem. Anal. Ed., 11, 346.

Stamberg O. E., Theophilus D. R., 1945. Photolysis of riboflavin in milk. J. Dairy Sci., 28, 269.

ZIEGLER J. A., 1944. Photochemical destruction of vitamine $\mathrm{B}_{2}$ in milk. J. Amer. Chem. Soc., 66, 1039.

\title{
L'UTILISATION DE COLORANTS POUR DÉCELER LA PRÉSENCE D'ANTIBIOTIQUES DANS LE LAIT (1)
} (fin)

\author{
par \\ Professeur Docteur P. KästLI (Suisse)
}

\subsection{Essais effectués par nos soins [24].}

Afin de pouvoir former notre propre opinion sur les effets produits par les additions de colorants, nous avons procédé dans notre Institut à quelques essais qui devront être étendus par la suite.

\subsubsection{Essais au moyen de chlorophylle.}

Deux vaches ont été traitées en même temps chacune sur deux quartiers à l'aide d'une préparation contenant $100000 \mathrm{U}$ de pénicilline $\mathrm{G}$ sous forme de sel de potassium, 0,4 g d'une solution huileuse de chlorophylle, ainsi que de l'oleum sésame et du stéarate d'aluminium pour $10 \mathrm{ml}$. On a obtenu les résultats suivants :

(1) Bulletin F.I.L. 1963. VI (Rapport présenté par le $\mathrm{P}^{\mathrm{r}} \mathrm{D}^{\mathrm{r}} P$. Kästli et reproduit avec l'autorisation de la Fédération Internationale de Laiterie). 


\begin{tabular}{|c|c|c|c|c|c|c|}
\hline \multirow{3}{*}{$\begin{array}{l}\text { Traites } \\
\text { après traitement }\end{array}$} & \multicolumn{2}{|c|}{ Quantité de lait en $\mathrm{kg}$} & \multicolumn{2}{|c|}{$\begin{array}{c}\text { Pénicilline U } \\
\text { par ml de lait (1) }\end{array}$} & \multicolumn{2}{|c|}{ Coloration } \\
\hline & \multicolumn{6}{|c|}{ Vache } \\
\hline & I & II & I & II & I & II \\
\hline $1 \ldots \ldots \ldots \ldots$ & 3,1 & 3,0 & 100,0 & 12,0 & +++ & + \\
\hline $2 \ldots \ldots \ldots \ldots$ & 3,2 & 3,3 & 50,0 & 5,0 & ++ & +- \\
\hline $3 \ldots \ldots \ldots$ & 3,0 & 3,0 & 2,0 & 0,9 & +- & - \\
\hline $4 \ldots \ldots \ldots \ldots$ & 3,2 & 3,3 & 0,5 & 0,5 & - & - \\
\hline $5 \ldots \ldots \ldots \ldots$ & 2,2 & 2,9 & 0,07 & 0,05 & - & 一 \\
\hline $6 \ldots \ldots \ldots$ & 3,1 & 3,4 & 0,03 & 0,02 & 一 & - \\
\hline
\end{tabular}

(1) Ensemble des traites provenant de quatre uuartiers.

Le résultat aberrant de cette expérience peut s'expliquer par le fait que nous avons utilisé seulement $0,4 \mathrm{~g}$ de chlorophylle huileuse au lieu de 2 ou $3 \mathrm{~g}$. Dans une lettre personnelle, KosikowskI me fit savoir qu'avec une dose plus élevée de chlorophylle, on peut s'attendre à une coloration du lait pendant 6 traites.

\begin{tabular}{|c|c|c|c|c|c|c|c|c|c|c|}
\hline \multirow{3}{*}{$\begin{array}{l}\text { Traites } \\
\text { après } \\
\text { traitement }\end{array}$} & \multicolumn{2}{|c|}{$\begin{array}{c}\text { Quantité } \\
\text { de lait } \\
\text { en kg }\end{array}$} & \multicolumn{2}{|c|}{$\begin{array}{l}\text { Unités de } \\
\text { pénicilline } \\
\text { par ml } \\
\text { de lait (1) }\end{array}$} & \multicolumn{2}{|c|}{$\begin{array}{r}\text { Indice de } \\
\text { visibilité (2) }\end{array}$} & \multicolumn{2}{|c|}{$\begin{array}{c}\text { Pénicilline } \\
\text { par indice (3) }\end{array}$} & \multicolumn{2}{|c|}{$\begin{array}{l}\text { Coloration } \\
\text { dans l'échan- } \\
\text { geur d'ions (4) }\end{array}$} \\
\hline & \multicolumn{10}{|c|}{ Vaches } \\
\hline & I & II & I & II & I & II & I & II & I & II \\
\hline $1 \ldots \ldots \ldots$ & 4,2 & 3,5 & 5,0 & 7,6 & 60 & 70 & 0,08 & 0,1 & & \\
\hline $2 \ldots \ldots \ldots$ & 3,1 & 2,7 & 1,0 & 0,8 & 10 & 14 & 0,1 & 0,06 & & \\
\hline $3 \ldots \ldots \ldots$ & 4,0 & 3,7 & 0,6 & 0,25 & 7 & 9 & 0,08 & 0,03 & & \\
\hline $4 \ldots \ldots \ldots$ & 3,1 & 2,6 & 0,15 & 0,1 & 3 & 5 & 0,05 & 0,02 & & \\
\hline $5 \ldots \ldots \ldots$ & 4,1 & 3,2 & 0,05 & 0 & 1 & 1 & 0,05 & - & & \\
\hline $6 \ldots \ldots \ldots$ & 3,4 & 3,0 & 0,02 & 0 & 0 & 0 & & & +++ & \\
\hline $7 \ldots \ldots \ldots$ & 4,2 & 3,6 & 0 & 0 & 0 & 0 & & & - & \\
\hline
\end{tabular}

(1) Ensemble des traites provenant de quatre quartiers.

(2) Degré de visibilité : l est encore visible à l'œil nu.

(3) Teneur en pénicilline en U par ml par degré de l'indice.

(4) Tube échangeur d'ions d'aprè Dalgaard, Mikkelsen et Rasmussen (2). 


\subsubsection{Essai avec du vert de lissamine.}

La préparation contenait $100000 \mathrm{U}$ de pénicilline, $0,10 \mathrm{~g}$ de colorant, de l'oleum sésame et du stéarate d'aluminium pour $10 \mathrm{ml}$. On traita également deux vaches à raison d'un quartier chacune.

Les vaches sur lesquelles on a effectué des expériences ne présentaient aucun symptôme de mammite. En résumé, nous avons également eu l'impression, à la suite de ces essais, que l'addition de vert de lissamine donnait des résultats plus favorables que l'addition de chlorophylle.

\section{III. - Avis des autorités relativement à l'addition de colo- rant à des antibiotiques en vue de traitements intra- mammaires}

Il ressort des réponses que nous ont faites les spécialistes qui ont procédé à des essais dans divers pays, que jusqu'à présent, la coloration des antibiotiques en vue de traitements intramammaires n'a été officiellement reconnue, autorisée ou prescrite dans aucun pays. Par ailleurs, l'industrie pharmaceutique n'a pas entrepris jusqu'à présent la fabrication de ces préparations sur une grande échelle et sur une base commerciale.

Aux Etats-Unis, d'après des renseignements fournis par Kosikowski (15), seule une approbation de principe a pu être obtenue de la "Food and Drug Administration (FDA)" pour des colorants inoffensifs du point de vue physiologique, pour autant qu'ils soient détectables dans le lait jusqu'à 1 ppm (cette condition était remplie dans les essais effectués avec le vert de lissamine). Toutefois, il n'existe encore aucune autorisation officielle pour des préparations colorées.

D'après une communication de HARGROve (11), la FDA est plutôt opposée à l'emploi de la fluorescéine en raison de sa parenté avec les colorants dérivés du goudron. Il en est de même de la chlorophylle fluorescente.

ShaHANi (21) a signalé, en réponse à notre question, qu'une demande avait été adressée à la FDA tendant à prescrire la coloration des antibiotiques utilisés pour des traitements intramammaires. Par contre, l'utilisation du bleu acide brillant V.S. n'a pas dépassé le stade expérimental.

Au Danemark non plus, selon les indications fournies par Dalgaard-Mikkelsen (3), l'emploi du vert de lissamine $n^{0} 4$ n'est pas encore généralisé en ce qui concerne les préparations 
d'antibiotiques. Néanmoins, on a officiellement autorisé l'addition aux aliments de ce colorant qui peut ainsi être utilisé sans difficulté pour la coloration des antibiotiques.

D'après Frank (7), on s'est borné jusqu'à présent en Allemagne, à exiger pour l'application d'antibiotiques destinés à des traitements intramammaires, l'élimination du lait pendant cinq jours. Les services compétents n'ont cependant pas encore examiné la question de l'addition d'un colorant.

Ayant constaté que $0,6-7,5$ p. 100 du lait livré à la laiterie de Weihenstephan contenaient des antibiotiques, un questionnaire fut adressé à 382 laiteries leur demandant quelles méthodes elles recommandaient pour empêcher la livraison de lait contenant des antibiotiques. Des réponses parvenues, il ressort que 50 p. 100 proposèrent la coloration des antibiotiques, 7 p. 100 l'obligation pour les vétérinaires d'informer les laiteries quand les animaux avaient subi un traitement par des antibiotiques, 6 p. 100 l'établissement d'un contrôle régulier de la teneur du lait en antibiotiques, 37 p. 100 s'abstinrent de toute proposition (8).

En Australie, les opinions sur l'utilisation de colorants avec les antibiotiques pour les traitements intramammaires divergent. Le "Australian Agriculture Council " se prononce pour l'emploi de colorants, tandis que l'"Australian National Medical Council" ne l'approuve pas. D'autre part, le "Victorian Department of Health" a décidé de rendre légal l'emploi des colorants à partir du $1^{\text {er }}$ septembre $1962(6)$.

En Suisse, la Commission du lait a demandé aux autorités responsables d'envisager, en liaison avec l'industrie pharmaceutique, la possibilité de rendre obligatoire la coloration des antibiotiques destinés aux traitements intramammaires. Une firme de produits pharmaceutiques fabrique déjà des préparations de ce genre en liaison avec la Station Fédérale de Recherche Laitière.

\section{IV. - L'emploi de colorants au point de vue de l'hygiène alimentaire}

Il n'est guère vraisemblable que du lait dont la coloration est perceptible à l'œil nu soit livré au commerce. En effet, le fournisseur doit s'attendre à ce qu'un tel lait soit refusé et même, éventuellement à ce que sa fourniture donne lieu à des sanctions. A cet égard, le consommateur est protégé contre le risque d'absorber des colorants.

Même si cela devait exceptionnellement se produire, les quantités de colorant seraient si réduites qu'il n'y aurait rien à craindre. 
C'est pourquoi il ne faut pas attacher trop d'importance à la question de la toxicité des colorants.

Toutefois, compte tenu de la haute valeur nutritionnelle du lait, il conviendrait de s'en tenir au principe selon lequel le lait ne doit contenir aucune substance toxique, même si celle-ci, en raison de sa très faible concentration, n'est pas nuisible à la santé.

La coloration des antibiotiques peut se justifier par le fait que la présence de colorants dans le lait pose un problème d'hygiène beaucoup moins important que l'absorption des antibiotiques eux-mêmes. Si l'on réussit grâce à l'addition de colorants aux antibiotiques à limiter dans une large mesure ou même à éviter la fourniture de lait contenant des antibiotiques, on aura accompli sur le plan de l'hygiène alimentaire un progrès tel que l'on pourra accepter un risque d'absorption de colorants, risque qui ne peut être complètement exclu. Les effets nuisibles des antibiotiques sur le consommateur de lait (allergies) sont beaucoup plus graves que l'absorption possible de colorants qui, pratiquement, ne se produira pas.

Il est certain qu'il faut particulièrement tenir compte des allergies, quand des personnes sensibilisées à l'action de la pénicilline et de la streptomycine boivent du lait qui en contient.

Du point de vue de la toxicité, on ne pourra élever des objections qu'à l'endroit de la fluorescéine. La chlorophylle est connue comme étant absolument inoffensive, car il s'agit d'une substance se trouvant également dans les aliments végétaux à l'état naturel. En ce qui concerne les colorants dérivés du triphénylméthane, il convient de noter que, dans de nombreux pays, il est permis de les ajouter aux aliments.

Les essais effectués pendant deux ans sur des rats avec du vert de lissamine, ont montré que ce produit n'était pas cancérigène. Cependant, si on procède à des injections sous-cutanées des matières colorantes, on constate qu'elles ont un certain effet cancérigène. Celui-ci est produit par les trois colorants cités, mais c'est avec le vert de lissamine qu'il est le plus faible (2).

\section{V. - L'emploi des colorants du point de vue de l'économie laitière}

La question essentielle qui se pose est celle de savoir si le lait est suffisamment coloré lorsque la concentration des antibiotiques tient encore en échec les bactéries lactiques. Il importe peu, à cet égard, que la réduction de la concentration des colorants ou des antibiotiques, soit le résultat de la dilution au moyen de lait normal ou des traites qui suivent le traitement. 
Mais il faut également s'attendre à ce qu'un trayeur ait des scrupules à ajouter au lait normal un lait dont la coloration est visible, et cela même si du fait de l'addition à un lait de mélange, la modification de teinte est fortement réduite ou même si la teinte disparaît complètement. Il ne peut pas savoir avant de verser un lait individuel coloré dans un bidon ou dans un tank si la coloration sera encore visible. Il ne voudra probablement pas courir le risque que la totalité de son lait lui soit refusée. Quoi qu'il en soit, on peut estimer qu'en pratique l'addition de lait contenant des antibiotiques et provenant de différents troupeaux ne se produira que dans les cas où la concentration de l'antibiotique ou du colorant sera très faible.

Les expériences auxquelles on a procédé jusqu'à présent, ont montré que la coloration du lait au moyen de colorants du groupe du triphénylméthane peut atteindre une concentration de 0,05 à $0,1 \mathrm{U}$ de pénicilline par $\mathrm{ml}$ de lait. Si l'on utilise la méthode Dalgaard-Mikkelsen et Rasmussen, il est même possible de déceler au moyen d'une résine échangeuse d'ions la pénicilline colorée à l'aide du Vert de lissamine jusqu'à une concentration de $0,002 \mathrm{U}$ par ml.

Selon les indications de Storgards (20), les bactéries lactiques sont totalement utilisées lorsque la concentration de la pénicilline est de 0,05 à $0,5 \mathrm{U}$ par $\mathrm{ml}$ dans le cas des streptocoques, 0,05 à 5,0 U par ml pour les lactobacilles, 1,6 U par ml pour les leuconostocs.

La limite d'appréciation de la coloration du lait coïncide ainsi approximativement avec les valeurs marginales inférieures auxquelles l'acidification est inhibée. Si l'on traitait exclusivement du lait provenant de vaches ayant subi un traitement aux antibiotiques contre la mammite, il pourrait arriver que la concentration en antibiotiques soit telle qu'il ne soit plus possible de la déceler au moyen d'une coloration et qu'en conséquence le lait ne soit pas éliminé des fabrications. Toutefois, de tels cas ne se rencontrent guère en pratique. Normalement, le lait provenant de différents troupeaux est mélangé, avant la transformation, avec celui d'autres régions. Lorsque le lait est fourni en bidons, chaque bidon contient un mélange du lait de dix vaches au maximum. Même lorsque le lait est livré en citernes, il faut s'attendre à ce qu'il y ait un mélange avec du lait normal provenant d'autres citernes. C'est pourquoi, si on livrait du lait contenant de 0,05 à $0,1 \mathrm{U}$ de pénicilline par $\mathrm{ml}$ et dans lequel il ne serait plus possible de distinguer une coloration à l'œil nu, il faudrait compter sur un mélange avec du lait normal avant la transformation. Il suffit que la dilution au moyen de lait 
normal soit supérieure à $1 / 10$ pour rendre impossible toute perturbation de l'acidification.

Selon les indications de DalgaARd-Mikkelsen (2), du lait dont la coloration est encore visible à l'œil nu colore également le lait écrémé, le babeurre, le fromage et le lactosérum, mais ni le beurre, ni la crème.

La transformation en produits laitiers de laits dont la coloration est visible est peu probable, car si de tels laits étaient livrés, ils seraient refusés à la réception. Nous devons cependant examiner la question de savoir si un taux de colorant non perceptible à l'œil nu lors de la livraison du lait, pourrait entraîner une coloration des produits laitiers. Cela peut se produire plus spécialement lors de la transformation d'un tel lait en fromage ou en poudre de lait. Rien de précis n'a été publié à ce sujet et ce problème demande encore à être étudié de plus près. C'est surtout sur la fabrication des poudres de lait que des investigations devraient être faites.

Par ailleurs, il est assez intéressant de se demander si, lorsque des veaux et des porcs sont nourris avec du lait coloré, leur viande est également colorée. Cette question revêt une importance pratique surtout parce que les propriétaires d'animaux doivent être en mesure d'utiliser encore ce lait coloré d'une manière ou d'une autre, et son emploi comme aliment est la première solution à envisager.

Les investigations auxquelles DALGAARD-MIKKELSEN a procédé lui ont permis d'observer ce qui suit :

Lorsqu'on utilise, pour nourrir des veaux ou des pores, du lait fortement coloré, on ne décèle aucune coloration de la viande. Par contre, la muqueuse intestinale se colore temporairement. L'utilisation du lait coloré ne devrait donc pas susciter de difficultés dans les fermes où on se livre à l'élevage des porcs ou des veaux.

\section{VI. - Conclusions}

L'addition de colorants à des antibiotiques en vue du traitement intramammaire constitue à l'heure actuelle, dans les pays où il n'est pas possible d'établir un contrôle régulier des antibiotiques du lait et où un pourcentage relativement élevé de lait contenant des antibiotiques est livré, la mesure la plus efficace pour prévenir la mise en vente de lait contenant des antibiotiques.

WILson (22) écrit à ce sujet: "Il est probable que le moyen le plus efficace serait d'ajouter des colorants aux antibiotiques et il est certain que cette mesure aurait pour effet d'éliminer plus de 90 p. 100 du lait actuellement envoyé aux centres de ramassage. " 
Les matières colorantes qui se sont révélées les plus satisfaisantes sont les dérivés du triphénylméthane: vert de lissamine, bleu acide brillant V.S. et alphazurine F.G. Ils sont irréprochables du point de vue de l'hygiène alimentaire et supérieurs aux autres substances examinées jusqu'à présent quant à la coloration et quant à leur élimination parallèlement aux antibiotiques.

Dans l'intérêt général, il y a lieu d'empêcher la livraison de lait contenant des antibiotiques, aussi bien parce qu'il peut être nuisible à la santé (allergies) que pour des raisons de qualité de lait et des produits laitiers.

A cet égard, les différents pays devraient prendre toutes dispositions utiles et envisager la possibilité d'ajouter un colorant convenablement choisi aux antibiotiques destinés aux traitements intramammaires.

\section{BIBLIOGRAPHIE}

[1] Sv. Dalgaard-Mikkelsen et F. Rasmussen. Nord. Vet. Med,, 9, 852-854, (1957).

[2] Sv. Dalgaard-Mikkelsen et F. Rasmussen. $X V I^{\mathrm{e}}$ Congr. Int. Lait. VIII, 2, 465-473, (1962).

[3] Sv. DalgaARd-Mikkelsen. Briefiche Mitteilung (17-11-1961).

[4] D. J. Dawson et J. T. Fegan. Australia J. Dairy Techn., 15, 160-172 (1960).

[5] D. J. Dawson. Briefiche Mitteilung (11-10-1961).

[6] D. J. Dawson. Briefliche Mitteilung (6-7-1962).

[7] H. Frank. Briefliche Mitteilung (29-1-1962).

[8] H. Frank. Deutsche Molkerei Zeit, 83, 1162 (1962).

[9] R. E. Hargrove., R. J. Lehman et C. A. Mattews. J. Dairy Sci., 41, 617-623 (1958).

[10] Hargrove R. E., Plowman R. D. et Wright W. W. J. Dairy Sci., 42, 202-206, (1959).

[11] R. E. Hargrove. Briefiche Mitteilung (9-10-1961).

[12] P. Hogh et F. Rasmussen. Acta Vet. Scandinavica, 2, 185-197 (1961).

[13] P. Hogh et F. Rasmussen. Acta Vet. Scandinavica, 3, 51-64 (1962).

[14] F. V. KosIkowski, J. Milk et Food Techn., 23, 285-287 (1960).

[15] F. V. Kosikowski. Briefliche Mitteilung (9-10-1961).

[16] G. OBIGER Milchwissenschaft, 16, 359-364 (1961).

[17] F. Rasmussen et Simesen Birgitte. Nord. Vet. Med. 12, 120-132 (1960).

[18] K. M. Shahani, J. Milk et Food Techn. 24, 138-142 (1961).

[19] K. M. Shahani Antibiotics Annual, 1958/59. Med. Encycl. New York, 883-889.

[20] K. M. Shahani Milk Industry Foundation, 53rd Ann. Convention. nov. 2-4, 14-24 (1960).

[21] K. M. Shahani Briefliche Mitteilung (16-10-1961). 
[22] T., Smitasiri F. V. Kosikowski, R. S. Guthrie et M. G. Fincher. J. Milk et Food Techn., 21, 255-258 (1958).

[23] T. Storgards FIL-Bericht III-DOC 9;60/10 (Part. III-IDF Bulletin FIL-1962).

[24] Unveröffentliche Versuche Eidg. Milchwirtschaftliche Versuchsanstalt Liebefeld-Bern.

[25] C. D. WILson Dairy Engineering 78, 444-445 (1961).

\title{
REVUE
}

\section{L'INDUSTRIE LAITIERE DANS LE MONDE}

\author{
par \\ G. GENIN \\ Ingénieur E.P.C.I.
}

\section{GÉNÉRALITÉS}

\section{L'industrie laitière dans le Marché Commun}

Dans le cadre des différentes ententes qui doivent normaliser la position de l'agriculture dans les pays du Marché commun, il peut être intéressant de voir comment a évolué, de 1952 à 1962, la production du lait, et l'activité des différentes industries consommatrices. Voici quelques tableaux qui donnent une idée de cette évolution.

\begin{tabular}{|c|c|c|}
\hline \multirow[b]{2}{*}{$\begin{array}{l}\text { Lait de vache: } \\
\text { nombre de vaches laitières (en milliers). }\end{array}$} & 1952 & 1962 \\
\hline & 19 & 22192 \\
\hline teneur en graisse $\ldots \ldots \ldots \ldots \ldots \ldots$ & $36,2 \%$ & $37,3 \%$ \\
\hline production totale en $\mathrm{mi}$ & 37516 & 65458 \\
\hline ait de c & & \\
\hline nombr & 4246 & 2426 \\
\hline liers de tonnes .. & 1124 & 674 \\
\hline h & & \\
\hline nombre & 9382 & 7873 \\
\hline production totale en milliers de tonnes & 537 & 533 \\
\hline
\end{tabular}

D'autre part, l'évolution de la consommation a été la suivante : en fonction des différentes industries consommatrices en milliers de tonnes: 